\title{
Sensitivity Analysis of Carbon Nanotube Characteristics on Dynamic Behavior of Tapping-Mode Atomic Force Microscopy
}

\author{
M. H. Korayem*, N. Ebrahimi, S. Moloodi \\ Robotic Research Laboratory, Center of Excellence in Experimental Solid Mechanics and Dynamics, School of Mechanical Engineering, \\ Iran University of Science and Technology, Tehran, Iran \\ hkorayem@iust.ac.ir,n8_ebrahimi@iust.ac.ir
}

\begin{abstract}
Atomic force microscopy with attached carbon nanotube is an ideal tool in nano scale imaging, for its high precision caused by small tip diameter and high flexibility of carbon nanotube. In this paper sensitivity analysis of a tapping-mode atomic force microscopy (AFM) with large aspect ratio carbon nanotubes (CNT) probe tips is investigated. A finite-element method is used for vibration analysis of a continuous beam model of an AFM cantilever and vibration amplitude is extracted in different excitation frequencies and different tip separations from sample surface and the effect of nanotube characteristics such as its length, bending stiffness \& tilt angle on dynamic behavior of AFM is examined. The results show that decreasing the CNT length or increasing the CNT bending stiffness or the number of CNT layouts, increases the resonance frequency, but its effect is small. The results also show that using stiffer nanotubes removes the possibility of buckling in the range of the frequencies close the natural frequency of the cantilever. This can cause more stability due to less buckling. Sensitivity analysis helps better choosing of the carbon nanotube characteristics.
\end{abstract}

Keywords Carbon Nanotube, Tapping-mode Atomic Force Microscopy, Sensitivity Analysis

\section{Introduction}

Tapping-mode atomic force microscopy is an advanced method for imaging in nano scale. Carbon nanotubes were used as probe tips first by Dai et al[1]. They are potentially ideal atomic force microscopy probes due to their well-defined geometry, robust mechanical properties, and in the case of single-walled nanotubes (SWNTs), diameters approaching the size of small organic molecules[2]. The idea of using carbon nanotubes as atomic force microscopy tips has been recently concerned by the scientists because of their well characteristics and the less damage they cause to the sample. Besides the benefits of using carbon nanotubes as AFM tips there are some difficulties too. One of them is the hardness of attaching a CNT on the tip of an AFM and the other major problem is the little information about the dynamic and static behavior of nanotubes and the effects they cause on the dynamics of AFMs. Some scientists, using simple models, have analysed the dynamic behavior caused by the CNTs, some have done experiments and some other have studied other problems concerning using the CNTs.

* Corresponding author:

hkorayem@iust.ac.ir (M. H. Korayem)

Published online at http://journal.sapub.org/nn

Copyright (C) 2012 Scientific \& Academic Publishing. All Rights Reserved
For example Chen et al elucidated fundamental factors that determine AFM imaging resolution using experiments with SWNT probes and numerical simulations[2]. Raman et al investigated imaging artefacts caused by adherence of CNT to the wall of slopes of the sample surface and its effect on the resolution of the resultant images[3]. E. Lee offered a several degrees of freedom model for controlling of an AFM with parallel carbon nanotubes[4]. Lee et al offered a theoretical model using Euler-elastic beam theory for the CNT connected to the probe tip and investigated the theoretical and experimental results[5].

For studying the dynamic behavior of an AFM with an attached nanotube on the tip, S. I. Lee et al, investigated non-contact CNT tipped AFM by some experiments. In their experiments, the frequency response and the amplitude-separation data for a high aspect ratio multiwalled carbon nanotube (MWCNT) were extracted. Higher harmonics, corresponding to attractive and repulsive regimes, were measured versus frequency ranges when the CNT buckles dynamically. Surface imaging was done using MWCNT tip on $\mathrm{SiO} 2$ surface for validation of the imaging instabilities during the time the CNT was buckling[6]. Also S. I. Lee et al investigated nonlinear dynamics of an AFM with MWCNT tip experimentally and theoretically. They gave experimental nonlinear frequency response in tapping-mode[7]. 
C. V. Nguyen et al, presented some results about stability and lateral resolution of carbon nanotubes[8] and Snow et al, studied the factors which specify imaging stability with single-walled carbon nanotube (SWCNT) tipped AFMs. They used a numerical method for investigation of non-contact imaging and studied the effects of the vertical alignment of CNT with respect to the sample surface and also the CNT length on amplitude-separation diagram[9].

Some scientists have studied the friction of CNT on the surface for example, B. Bhushan et al carried out a comprehensive investigation of adhesion, friction and wear of a MWCNT tip, SWCNT tip and MWCNT arrays[10] while H. Lua et al quantitatively investigated sliding friction and adhesion properties of vertically aligned multi-walled carbon nanotube arrays and fluoro-nanodiamond films on glass substrate using AFM[11].

One major factor which affects the dynamics of nanotubes, is their bending and buckling behavior. H. W. Yap et al prepared some experiments with individual multiwall carbon nanotubes with different ranges of aspect ratios. The nanotubes were subjected to cyclic axial compression in large strains using atomic force microscopy. Distinct elastic buckling and post buckling phenomena were observed reproducibly and were ascribed to Euler, asymmetric shell buckling (i.e., kinking), and symmetric shell buckling[12].

For all advantages of carbon nanotubes, an investigation of CNT tipped AFM dynamics is considerable. As pointed researchers investigated CNT tipped AFM theoretically or experimentally for their stability, lateral resolution, imaging artefacts, buckling behavior and bifurcation or tried to offer an appropriate model for dynamic simulation and contact force between CNT \& sample surface, but obviously further investigation is still needed for clarifying CNT effect on dynamic behaviour of AFMs. Researchers have done experiments each with a special kind of carbon nanotube which helps investigation of CNT effect on AFM, but an appropriate sensitivity analysis of the effects of carbon nanotube on tapping-mode AFM has not been done.

In this paper sensitivity analysis of nanotube characteristics on tipping-mode AFM dynamics is noticed which was not done in the previous researches. It has been done theoretically and regarding to researchers' experimental analyses. Changing nanotube characteristics affect buckling behavior or critical buckling force, these factors besides AFM characteristics like its stiffness, excitation amplitude and frequency can change the behavior of Dynamic mode AFM. Dynamic mode AFM should be excited in its resonance frequency and attaching a nanotube on the tip of AFM changes the resonance frequency. Buckling resistance of the nanotube can make different changes on the resonance frequency or amplitude. Also the geometry of the CNT affects the imaging resolution, stability, the force transferred to the sample and in the result the damage which is caused on the sample. In this paper studying of these effects is attended. This knowledge will help an appropriate choice for the geometry and characteristics of nanotube.

\section{Theoretical Analysis}

An experiment was done for tip deflection versus tip-sample separation by[7] for an AFM with MWCNT tip. Their results showed that the complex nature of the tip-sample interaction at various separations can cause the MWCNT to buckle as well as slip, slide, and adhere to the surface of the sample depending on the tip-sample separation. These effects were demonstrated by measuring the static force-distance curve which records the force on the MWCNT tip as a function of the $\mathrm{z}$ travel (vertical) distance. As the tip approaches the surface, the cantilever first snaps into contact with the sample and then deflects linearly as the CNT tip pushes against the surface. After that the cantilever exhibits a nonlinear deflection by increasing $\mathrm{Z}$, indicating a buckling of the MWCNT tip and then near constant cantilever deflection indicates that the deformation is directed into MWCNT buckling rather than producing additional cantilever deflection. After $\sim 800 \mathrm{~nm}$ of indentation, there is a sudden decrease in cantilever deflection, suggesting that the MWCNT has slipped on the Highly Oriented Pyrolytic Graphite (HOPG) surface.

For analysing this system, first it is necessary to define the contact force applied on the cantilever. For simplification it is possible to assume that:

1- The CNT mass is infinitesimal compared to that of the probe, so the lateral inertia of the CNT is neglected.

2- The CNT deforms quasi-statically during tip-sample impact.

3- The effect of nanotube vibration is neglected, so it is assumed that in the same excitation frequency and tip separation, the CNT tip impacts the same point in each cycle.

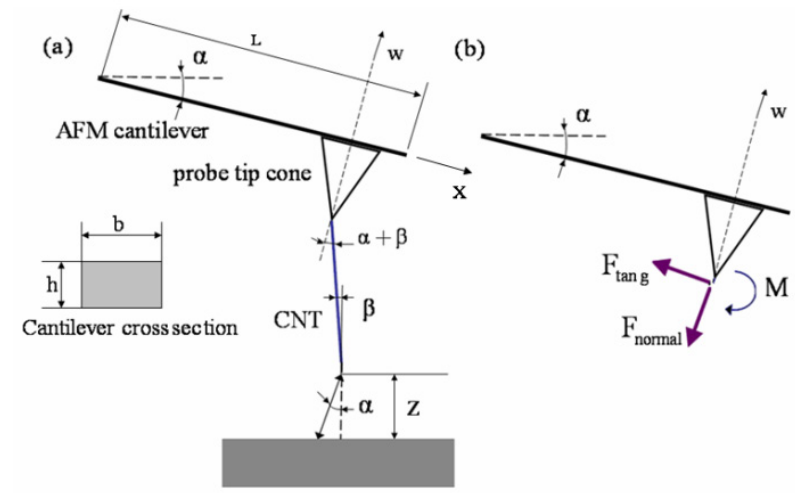

Figure 1. (a) Schematic diagram of AFM cantilever with CNT tip. The angle between probe and horizontal axis is $\alpha$ and the angle between CNT \& normal axis is $\beta$. Separation of CNT tip from sample is $z$. Lateral deflection of the probe is parallel to axis of $w$. (b) Diagram of the forces normal to the cantilever $\left(F_{\text {normal }}\right)$, tangent to the cantilever $\left(F_{\text {tang }}\right)$ and the moment $(M)$.

For defining the force applied on the cantilever, it is possible to simulate it as a function of distance[7].

1- In the separations larger than intermolecular distance (a0) the interaction force is carbon-carbon van der waals force $\left(F_{v d w}\right)$ which can be approximated as the van der waals force between a sphere with radius equal to CNT radius and a plane. 


$$
F_{v d w}=-\frac{H_{C-C} R}{6 z^{2}}
$$

Here $H_{C-C}$ is the carbon-carbon Hamakar constant, $R$ is the CNT tip radius and $z$ is transient tip-sample separation.

2- As the CNT tip approaches the surface, the repulsive interaction force increases and causes the CNT to be compressed and finally buckled $(z \leq 0)$. For calculating this buckle force, it is possible to use euler-elastica force equation which expresses the force as a function of deflection. In this paper, buckling force function is determined by working-model software, which gives normal and tangent forces and the moment. Figure 2 shows the schematic model made in working model for calculating forces and moment. If the CNT doesn't slide on the sample surface, it is possible to assume that the CNT tip is jointed on the sample surface and, in its other side, due to the vibration of the cantilever, it is sliding in a direction parallel to $\mathrm{w}$ axis, normal to the AFM cantilever (Fig. 1). We cause the CNT to buckle a little in different values and then save the values of the reaction forces and moment applied on the cantilever by the CNT, calculated by the software. These reaction forces are normal force $\left(F_{\text {normal }-C N T}\right)$, tangential force $\left(F_{\tan g-C N T}\right)$ and moment $\left(M_{C N T}\right)$. Finally diagram of the forces and moment versus buckling amount is obtained. These diagrams are approximately linear. Equations (2), (3), and (4) are linear approximations of the CNT force \& moment versus deflection curves. Figure 3 shows the linear approximated equations for these forces and the moment. It is important to mention that we let the nanotube to buckle just a little, because more buckling causes instability.

$$
\begin{gathered}
F_{n o r m a l-C N T}=-K_{v} z+f_{v} \\
F_{\tan g-C N T}=-K_{h} z+f_{h} \\
M_{C N T}=-K_{t} z+f_{t}
\end{gathered}
$$

$K_{v}, f_{v}, K_{h}, f_{h}, K_{t}$ and $f_{t}$ are the coefficients of the approximated linear equations for $F_{\text {normal }-C N T}, F_{\tan g-C N T}$ and $M_{C N T}$.

3- In the range $0<z \leq a_{0}$, the interaction normal force $\left(F_{N-M}\right)$ and tangential force $\left(F_{T-M}\right)$ are approximated as a quadratic polynomial which interpolates between force functions discussed above[7]. In this range moment is zero.

$$
\begin{aligned}
& F_{N-M}=-K_{v} z+f_{v}+\frac{1}{a_{0}^{2}}\left(\frac{H R}{6 a_{0}^{2}} \cos \alpha-\left(-K_{v} a_{0}+f_{v}\right)\right) \\
& F_{T-M}=-K_{h} z+f_{h}+\frac{1}{a_{0}^{2}}\left(\frac{H R}{6 a_{0}^{2}} \sin \alpha-\left(-K_{h} a_{0}+f_{h}\right)\right)
\end{aligned}
$$

Considering the angle between probe and horizontal axis $\alpha$ (figure 1), the interaction force can be expressed as below, the normal force $\left(F_{\text {normal }}(z)\right)$ is

$$
F_{n o r m a l}(z)=\left\{\begin{array}{lr}
F_{v d w}(z) \cos (\alpha) & a_{0} \leq z \\
F_{N-M} & 0<z<a_{0} \\
F_{\text {normal }-C N T} & z \leq 0
\end{array}\right.
$$

The tangential force $\left(F_{\text {tang }}(z)\right)$ is

$$
F_{\tan g}(z)=\left\{\begin{array}{lc}
F_{v d w}(z) \sin (\alpha) & a_{0} \leq z \\
F_{T-M} & 0<z<a_{0} \\
F_{\tan g-C N T} & z \leq 0
\end{array}\right.
$$

And the moment $(M(z))$ is

$$
M(z)=\left\{\begin{array}{lc}
0 & a_{0} \leq z \\
0 & 0<z<a_{0} \\
M_{C N T} & z \leq 0
\end{array}\right.
$$

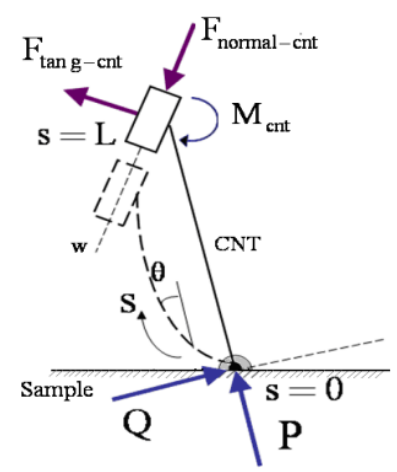

Figure 2. Schematic model made in working model for calculating forces and moment. $P \& Q$ are normal and tangential forces applied on CNT tip from sample. $F_{\text {normal-CNT }}, F_{\tan g-C N T}$ and $M_{C N T}$ are normal and tangential reaction forces $\&$ moment caused by $\mathrm{CNT}$ applied on the cantilever.

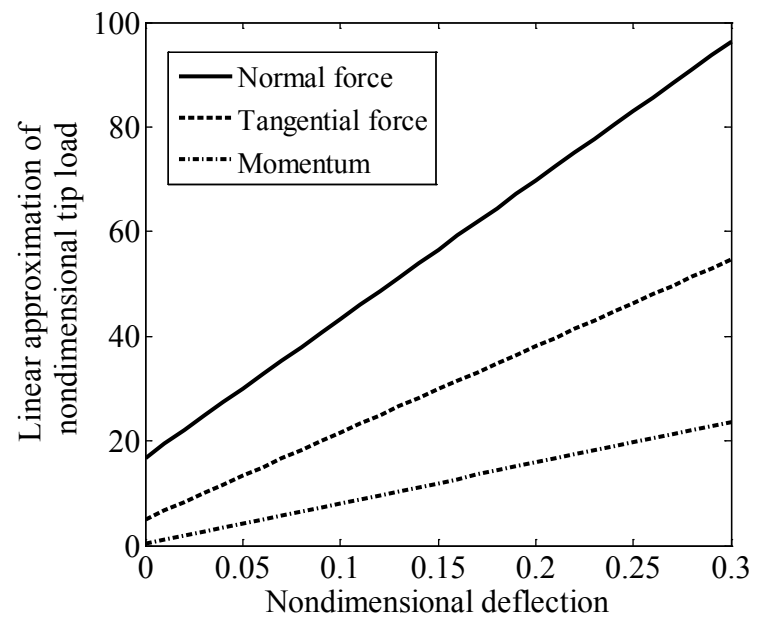

Figure 3. Approximated linear functions for normal force $\left(F_{\text {normal-CNT }} L^{2} / E I\right)$, tangential force $\left(F_{\tan g-C N T} L^{2} / E I\right)$ and moment $\left(M_{C N T} L / E I\right)$.

The governing equation for cantilever vibration is

$$
E I \frac{\partial^{4} w(x, t)}{\partial x^{4}}+c \frac{\partial w(x, t)}{\partial t}+\rho A \frac{\partial^{2} w(x, t)}{\partial t^{2}}=F(x, t)
$$

Where $w(x, t)$ is the cantilever lateral displacement, $E$ is the Young's modulus of the cantilever, $\rho$ is the mass density, $A$ is the cross section area of the cantilever, $I$ is the moment of inertia about lateral axis and $\mathrm{c}$ is the damping coefficient. The boundary conditions for the cantilever are $\left.w(x, t)\right|_{x=0}=0,\left.\quad w^{\prime}(x, t)\right|_{x=0}=0$, $\left.E I_{y} w^{\prime \prime}(x, t)\right|_{x=L}=0, \quad$ and $\left.\quad E I_{y} w^{\prime \prime \prime}(x, t)\right|_{x=L}=0$. 
$F(x, t)$ is the interaction force between the sample and cantilever which was discussed in equations (7), (8) \& (9).

For solving the governing equation with finite-element method the cantilever is divided by beam elements and the tip is modelled as a rigid cone. At each node of the beam element, there are two degrees of freedom, one is the translation displacement and the other is the rotation angle, and so the element nodal displacement vector is[13-14]

$$
W=\left\{w_{1}, \theta_{1}, w_{2}, \theta_{2}\right\}^{T}
$$

The corresponding element nodal force vector, consisting of shear force and moment at each node, is

$$
F=\left\{F_{1}, M_{1}, F_{2}, M_{2}\right\}^{T}
$$

For each element the vibration equation and the boundary conditions are expressed as Equations (13), (14), and (15) where $\mathrm{x}$ is the coordinate along the cantilever and Le is the length of the element[13].

$$
\begin{gathered}
w(x)=C_{1} x^{3}+C_{2} x^{2}+C_{3} x+C_{4} \\
\left.w(x, t)\right|_{x=0}=W_{1} \quad,\left.\quad w(L, t)\right|_{x=L_{e}}=W_{2} \\
\left.\frac{\partial w(x, t)}{\partial x}\right|_{x=0}=\theta_{1} \quad,\left.\quad \frac{\partial w(x, t)}{\partial x}\right|_{x=L_{e}}=\theta_{2}
\end{gathered}
$$

Here $C_{i}$ are

$$
\begin{aligned}
& C_{1}=\frac{1}{L_{e}^{3}}\left(2 W_{1}+L_{e} \theta_{1}-2 W_{2}+L_{e} \theta_{2}\right) \\
& C_{2}=\frac{1}{L_{e}^{2}}\left(-3 W_{1}-2 L_{e} \theta_{1}+3 W_{2}-L_{e} \theta_{2}\right) \\
& C_{3}=\theta_{1} / L_{e} \\
& C_{4}=W_{1}
\end{aligned}
$$

So $w(x, t)$ can be defined as the product of a vector $\mathrm{N}$ by the element displacement vector (Vector $\mathrm{N}$ is defined in the appendix).

$$
\begin{gathered}
w(x, t)=N W \\
\frac{\partial w(x, t)}{\partial t}=N \dot{W} \\
\frac{\partial w(x, t)}{\partial x}=\frac{\partial N}{\partial x} W
\end{gathered}
$$

The elemental mass, and stiffness matrixes can be defined as Equations (19) and (20).

$$
\begin{gathered}
M_{e}=\int_{0}^{L_{e}} \rho A\left(N^{T} N\right) d x \\
K_{e}=\int_{0}^{L_{e}} E I\left(\frac{\partial^{2} N^{T}}{\partial x^{2}}\right)\left(\frac{\partial^{2} N}{\partial x^{2}}\right) d x
\end{gathered}
$$

The governing equation for the beam element can be written in matrix form as:

$$
M_{e} \ddot{W}+K_{e} W=F_{e}
$$

$F_{e}$ is the applied force vector on each element. By assembling the mass and elasticity matrixes, the total equations can be found. The final matrix will be a $(2 n+2) *(2 n+2)$ matrix. The force vector is zero in each node except for the first node and the node that the tip is connected. The forces and mo- ment in the node of the tip is the interaction forces and moment between probe and sample surface which was discussed before. The cantilever is excited by a piezoelectric exciter by a frequent voltage in the first node. The piezoelectric has a great mass in comparison to the cantilever, so the vibration inertial forces of the cantilever are negligible in comparison to the inertia of the piezoelectric holder. So when the holder is excited with a frequent voltage and a constant amplitude (Asp), it causes the cantilever to vibrate by the holder with a constant amplitude. Thereby for the first node we have

$$
\begin{gathered}
w_{1}=A_{s p} \sin (\omega t) \\
\theta_{1}=0
\end{gathered}
$$

Here $\omega$ is the excitation frequency. Then we should convert the equations into standard form of finite element equations. Because $\theta=0$, the second row and second column of the matrixes can be omitted and the first row can be separated from the total matrix. The first row of the matrix is the equation from which F1 can be found and we don't need, so the equation belongs to the first row of the matrix, can be omitted from the total equations. The term belonging to $\left\{k_{31}, k_{41}, k_{51}, \ldots.\right\} w_{1}$ is transferred to the right side of the equation and is added to the force vector. The same action is done for mass matrix too. So we have a $2 n * 2 n$ matrix equation as:

$$
\begin{aligned}
& {[K]_{2 n * 2 n}\left\{\begin{array}{c}
w_{2} \\
\theta_{2} \\
\vdots
\end{array}\right\}_{2 n}+[M]_{2 n * 2 n}\left\{\begin{array}{c}
\ddot{w}_{2} \\
\ddot{\theta}_{2} \\
\vdots
\end{array}\right\}_{2 n}=} \\
& \left\{\begin{array}{c}
F_{2} \\
M_{2} \\
\vdots
\end{array}\right\}_{2 n}-w_{1}\left\{\begin{array}{c}
k_{31} \\
k_{41} \\
\vdots
\end{array}\right\}_{2 n}-\ddot{w}_{1}\left\{\begin{array}{c}
m_{31} \\
m_{41} \\
\vdots
\end{array}\right\}_{2 n}
\end{aligned}
$$

The resultant standard form equations can be solved. The probe tip has a shape like a cone or a pyramid and of the same material of the cantilever. For adding the effect of the probe tip mass in the mass matrix, the probe tip is assumed as a connected rigid cone with the height $H$, mass $\mathrm{m}_{\text {tip }}$, density $\rho$, inertial moment about lateral axis passed from mass centre $I$, tip curvature radius of $\mathrm{R}_{\text {cone }}$, base circle radius of $\mathrm{r}$ and distance between mass centre and the cantilever of $\mathrm{H}_{\mathrm{mc}}$ and belonging to a node which is located in a distance from the end of the probe and with two degrees of freedom, vertical displacement and rotation. $\mathrm{H}_{\mathrm{mc}}$, mass and gyration radius about the lateral axis (gr) is[15].

$$
\begin{aligned}
& H_{m c}=H / 4 \\
& m_{t i p}=\pi R^{2} H \rho / 3 \\
& \bar{I}=3 / 20 m_{t i p} r^{2}+1 / 10 m_{t i p} H^{2} \\
& g r=\sqrt{\frac{\bar{I}}{m_{t i p}}}
\end{aligned}
$$

Now we write the equation for the equilibrium of the forces applied on the cone and calculate the moment about the mass centre (Figure 4). 


$$
\begin{aligned}
& -F_{V-t i p}+F_{v}=m_{t i p} \ddot{w} \\
& F_{H}-F_{H-t i p}=m_{t i p} \times H_{m c} \times \ddot{\theta} \\
& -T_{\text {tip }}+F_{H}\left(H-H_{m c}\right)+F_{H-t i p} H_{m c}=m_{t i p} \times g r^{2} \times \ddot{\theta}
\end{aligned}
$$

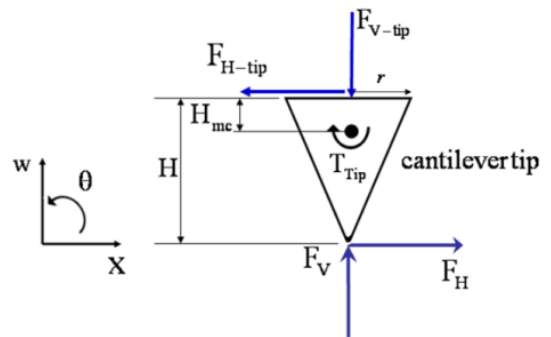

Figure 4. Free body diagram of the forces interacted on tip probe cone

In these terms, $F_{V}$ is the vertical interaction force, $F_{H}$ is the horizontal interaction force, $F_{V-t i p}, F_{H-t i p}, T_{t i p}$ are the reaction forces due to the vertical and horizontal forces and the moment applied on the tip probe cone. Because $\theta$ is small, other accelerations are negligible. By simplification, we get equations (27) \& (28) and then add the specified terms to mass matrix and force vector. $T_{t i p}$ and $F_{t i p}$ are two elements of force vector and in the fact are the external forces.

$$
\begin{aligned}
& F_{V-\text { tip }}=\underbrace{F_{v}}_{\begin{array}{l}
\text { Add to } \\
\text { force } \\
\text { vector }
\end{array}}-\underbrace{m_{\text {tip }}}_{\begin{array}{l}
\text { Add to } \\
\text { mass } \\
\text { matrix }
\end{array}} \text { w } \\
& T_{\text {tip }}=\underbrace{F_{H} H}_{\begin{array}{c}
\text { Add to } \\
\text { force } \\
\text { vector }
\end{array}}-\underbrace{\left(m_{\text {tip }} \times H_{m c}^{2}+m_{\text {tip }} \times g r^{2}\right)}_{\begin{array}{l}
\text { Add to } \\
\text { mass } \\
\text { matrix }
\end{array}} \ddot{\theta}
\end{aligned}
$$

With mass and stiffness matrixes, natural frequencies and shape modes can be found. In these equations, $\omega$ is the natural frequency and $\left\{\phi_{i}\right\}$ is the i'th shape mode vector and $\Phi=\left[\begin{array}{lll}\varphi_{1} & \cdots & \varphi_{2 n}\end{array}\right]$ is the shape mode matrix.

For accounting the effect of damping matrix, we have

$$
M \ddot{W}+C \dot{W}+K W=F
$$

If we set $W=\Phi P$, we can normalize the equations, by multiplying the transpose of the shape mode matrix $\left(\Phi^{-1}\right)$ into the left hand of the equation:

$$
\left[M_{\text {Diag }}\right]\{\ddot{P}\}+\left[C_{\text {Diag }}\right]\{\dot{P}\}+\left[K_{\text {Diag }}\right]\{P\}=\Phi^{T} F
$$

Where $\left[M_{\text {Diag }}\right],\left[K_{\text {Diag }}\right] \&\left[C_{\text {Diag }}\right]$ are diagonal mass, stiffness and damping matrixes. Each decoupled equation, is the equation of oscillation of an independent vibrator with natural frequency of $\omega_{n, i}$ and damping ratio of $\zeta_{i}$ which has the relation $\zeta=1 / 2 Q$ with quality factor $Q$.

$$
\ddot{P}_{i}+2 \zeta_{i} \omega_{n, i} \dot{P}_{i}+\omega_{n, i}^{2} P_{i}=0
$$

So the modal damping matrix is:

$$
C_{\text {Diag }}(i, j)= \begin{cases}\frac{\sqrt{m_{d, i} k_{d, i}}}{Q_{i}} & i=j \\ 0 & i \neq j\end{cases}
$$

$m_{d, i}$ and $k_{d, i}$ are the elements of the diagonal mass and stiffness matrix. So the Damping matrix is:

$$
C=\left(\Phi^{T}\right)^{-1} C_{\text {Diag }}(\Phi)^{-1}
$$

The equations can be solved by Newmark method. Shape function $\mathrm{N}$, Mass and stiffness element matrix and a brief discussion on Newmark method are been given in the appendix.

\section{Simulation and Results}

\subsection{Simulation}

For simulation and dynamic analysis, the values of the properties listed in table (1) are used[7, $16 \& 17]$. Figure 6 shows the frequency response of the simulation, which is validated with the results of experiments done in[7] (Fig. 5).

Table 1. The values of the properties used in simulation

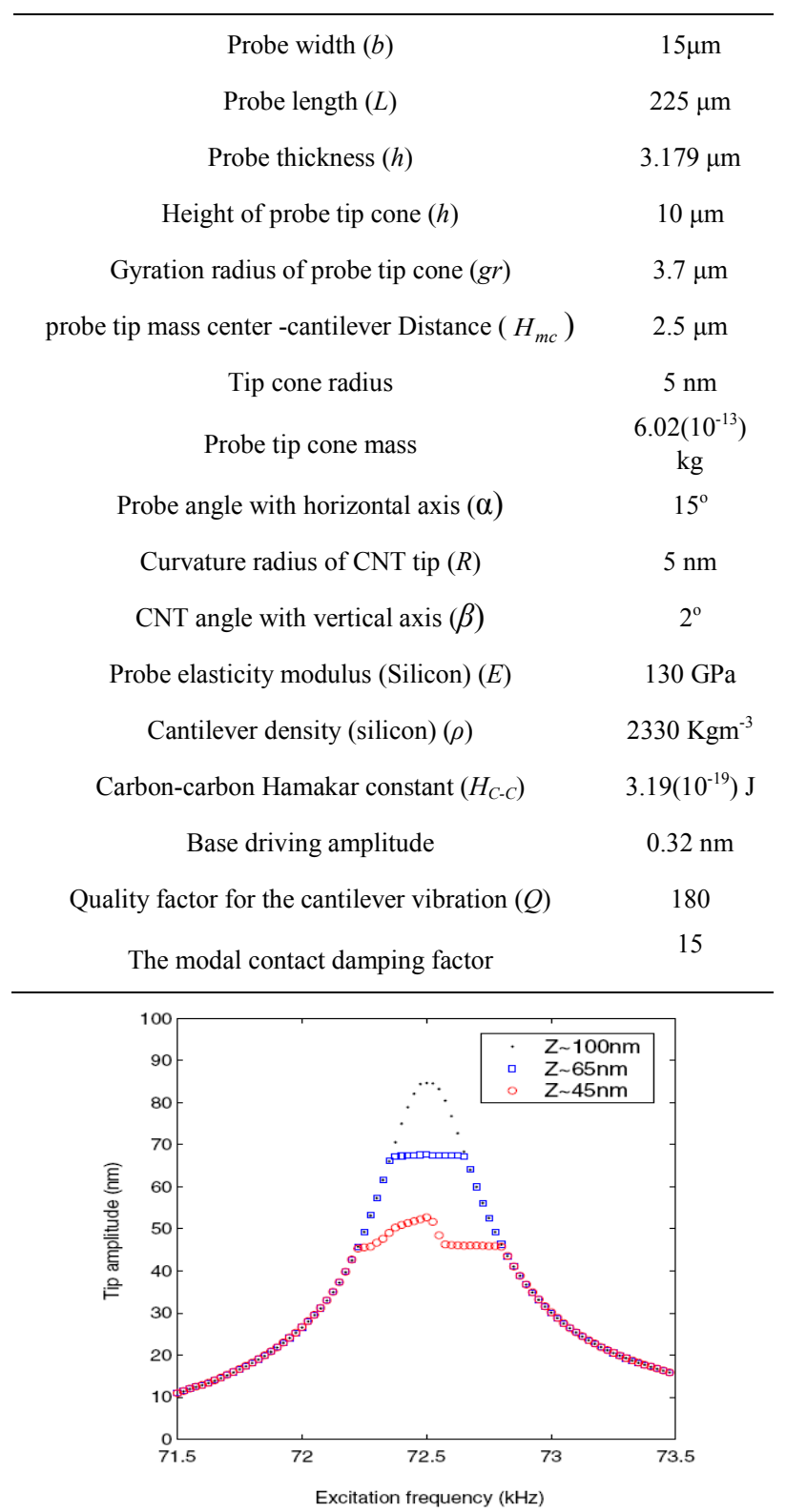

Figure 5. Experimental results of amplitude-frequency CNT tipped AFM on HOPG surface in different separations[7] 


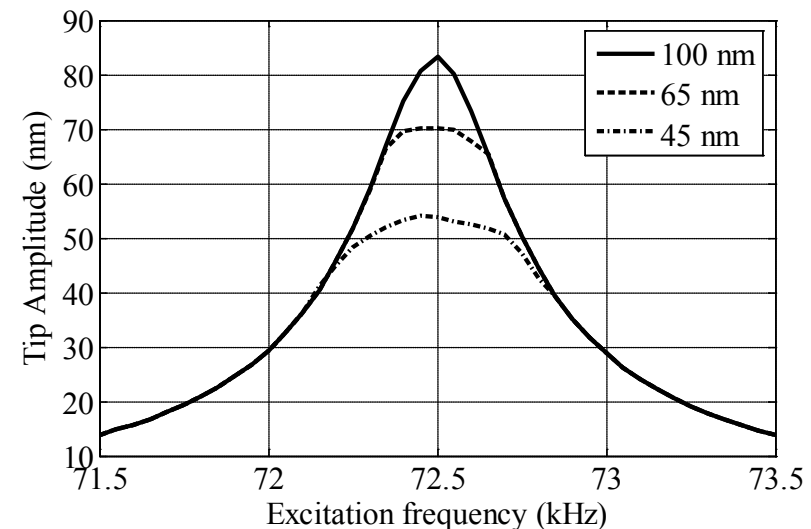

Figure 6. Simulated of amplitude-frequency in different separations by MATLAB

\subsection{Sensitivity Analysis}

Some Figures are extracted based on Euler-Elastica buckling force prediction by changing the CNT length, diameter or tilt angle or separation from surface. For this the buckle force introduced before $\left(F_{\text {normal }-C N T}, F_{\text {tan } g-C N T}\right.$ \& $M_{C N T}$ ) is made with the new parameters of CNT length, diameter or tilt angle and then this force is used for extracting the specified Figures. As theory of Euler-Elastica is proper for large aspect-ratio beams, we have used parameters in a range which have large aspect-ratio carbon nanotubes.

Figure 7 shows the amplitude-frequency diagram in constant separation of $45 \mathrm{~nm}$ with three different nanotube lengths. As what is shown in Figure 7, decreasing the CNT length causes increasing of resonance frequency just a little. The resonance frequency is $72.5 \mathrm{kHz}$. For the nanotube with $7500 \mathrm{~nm}$ length, the resonance is equal to $72.5 \mathrm{kHz}$ too. This means that the buckling resistance is very little. For shorter nanotubes the buckling resistance is increased and the resonance is increased a little. The nanotube can imagined as a spring which its stiffness enlarges the total stiffness of the AFM.

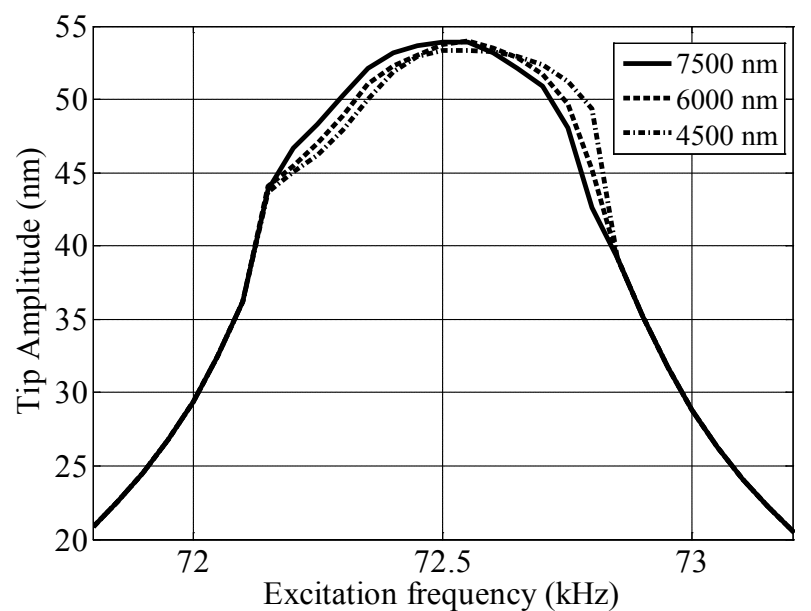

Figure 7. Simulation for amplitude-frequency diagram in constant separation of $45 \mathrm{~nm}$ with different nanotube lengths

Figure 8 , shows amplitude-frequency diagram in the constant separation of cantilever from the sample $(7545 \mathrm{~nm})$ with different nanotube lengths. Varying the CNT length causes the CNT tip separation changes. It is seen that decreasing the CNT length causes increasing the vibration amplitude. This shows that the cantilever separation from the sample is not important and just the CNT tip separation from sample surface determines the oscillation amplitude. Increasing the separation makes more freedom in the oscillation of the probe and so increases the oscillation amplitude.

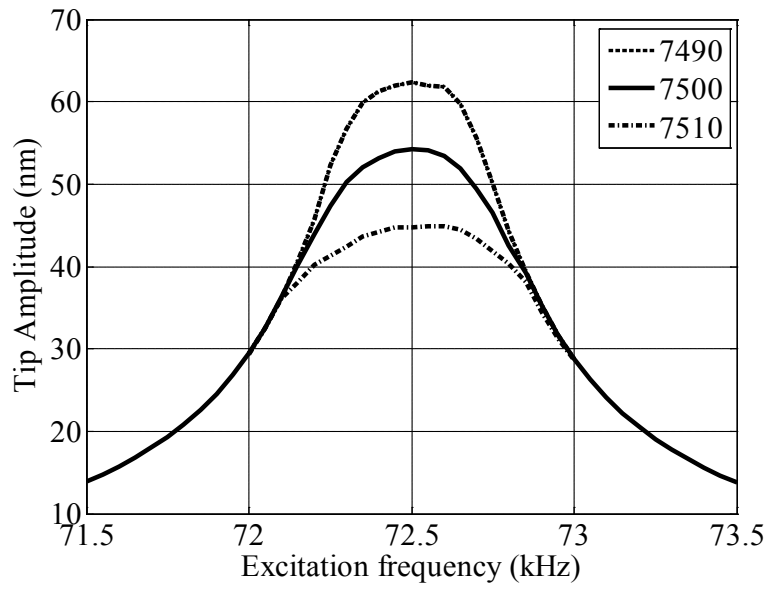

Figure 8. Simulation for amplitude-frequency diagram in the constant separation of cantilever from the sample $(7545 \mathrm{~nm})$ with different nanotube lengths

Figure 9 shows simulation for vibration amplitude versus separation from sample, with CNT length of $7500 \mathrm{~nm}$ and two different excitation frequencies of 72.4 and $72.5 \mathrm{KHz}$. Amplitude versus separation diagrams are important in the study of tapping mode AFMs, because they can represent the amplitude in different separations. In CNT tipped AFMs the amplitude is larger than the separation between the CNT tip and the sample and this is because of the buckling of the CNT.

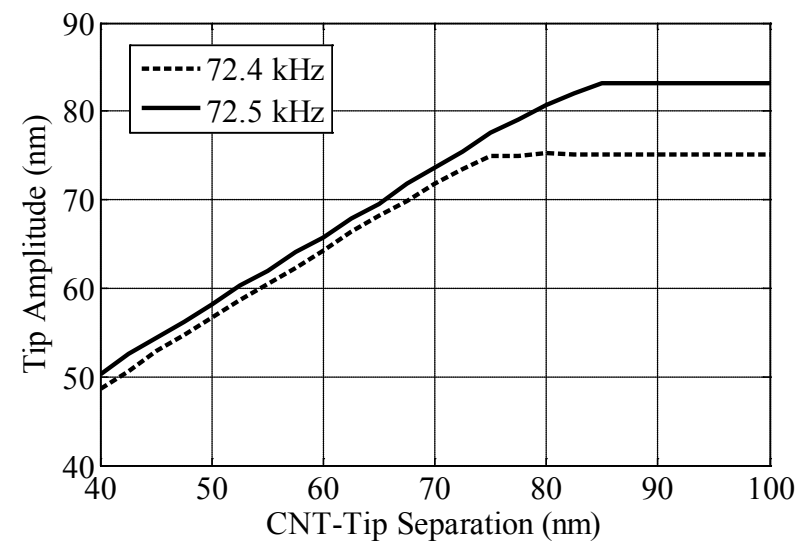

Figure 9. Simulation for vibration amplitude versus separation from sample, with CNT length of $7500 \mathrm{~nm}$ and different excitation frequencies

As conventional AFM (without CNT) increasing the separation causes increasing oscillation amplitude. Also after a specified separation the cantilever reaches to its maximum amplitude, but it is seen that in smaller separations the resonance amplitude in comparison with separation is larger. This means that in smaller separations the nanotube buckles 
more. The linear changes of the amplitude versus separation are seen in both excitation frequencies. In excitation frequency of $72.5 \mathrm{KHz}$, free amplitude vibration is obtained in farther separations related to the excitation frequency of 72.4 $\mathrm{KHz}$. Also the resonance amplitude is smaller. This can be noticed in the states when we don't like the CNT buckles much.

Figure 10 shows amplitude versus excitation frequency with different nanotube radiuses of 5, $7 \& 9 \mathrm{~nm}$ and $7500 \mathrm{~nm}$ length. Increasing the radius has caused increasing the resonance frequency. In other words increasing the diameter of the nanotube increases total stiffness of the AFM and this increases the resonance frequency.

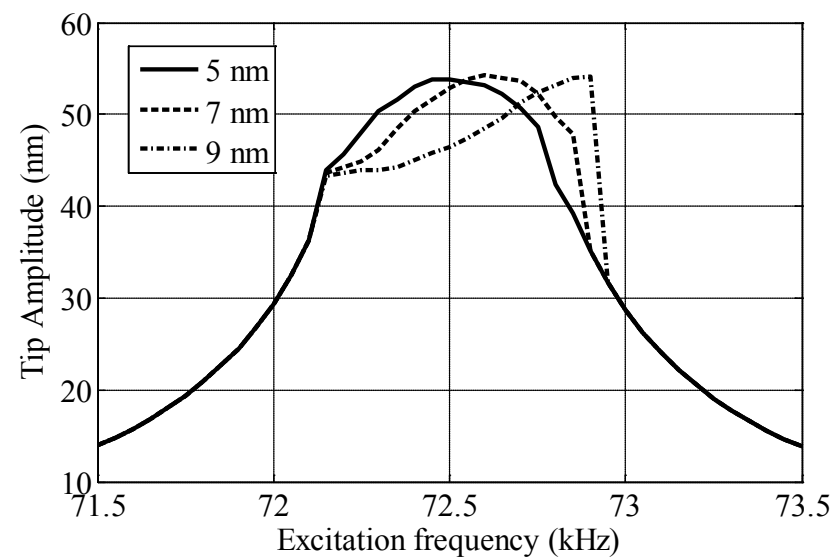

Figure 10. Simulation for amplitude versus excitation frequency with different nanotube diameters and $7500 \mathrm{~nm}$ length

Figure 11 shows the frequency response for different angles between nanotube and vertical axis. It is shown that changes of beta in small values don't have a considerable effect on resonance frequency and vibration amplitude. By increasing beta, the vibration amplitude is increased a little. Larger attachment angle can cause the nanotube to buckle easier.

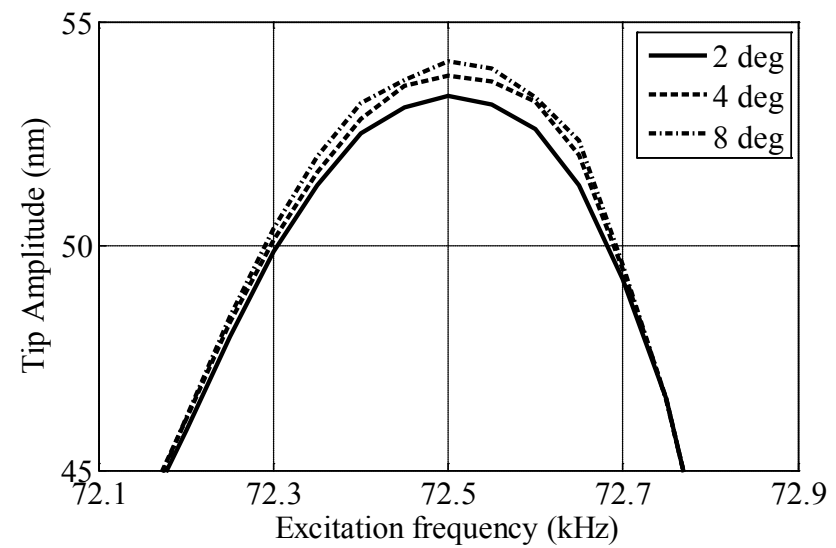

Figure 11. Simulation for frequency response for different angle between nanotube and vertical axis in separation of $45 \mathrm{~nm}$

Figure 12 shows simulation of the frequency response for a nanotube with length of $4000 \mathrm{~nm}$ and radius of $10 \mathrm{~nm}$. It is seen that using stiffer nanotubes removes the possibility of buckling in the range of frequencies around natural fre- quency of the cantilever. This can reduces the instability due to the buckling of the nanotube, although more force is transferred to the sample. This situation is ideal in the cases where we need a high aspect ratio tip and we don't want the instabilities caused by the buckling of the nanotube.

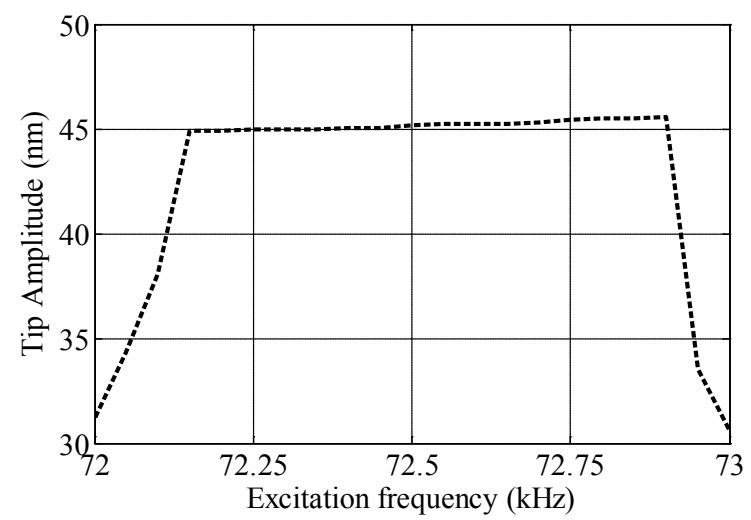

Figure 12. Simulation for frequency response for a nanotube with length of $4000 \mathrm{~nm}$ and radius of $10 \mathrm{~nm}$

Totally the diagrams represent that vibration amplitude is more affected by the amount of excitation force (free amplitude vibration) and separation between CNT tip and the sample. Cantilever separation from sample is not so effective. Decreasing the CNT length, causes increasing of buckling critical force and increasing of the resonance frequency. Increasing the CNT diameter, causes increasing the resonance frequency. The CNT angle is not so effective in small ranges of beta. Amplitude versus separation diagram shows the linear amount of buckling versus separation.

Choice of CNT characteristics should be done regarding to buckling stiffness. Buckling stiffness may follow different rules for different nanotubes, nanotubes with larger aspect ratios, have a treatment like Euler buckling rule, but nanotubes with smaller aspect ratios may have shell buckling which makes a different behavior from Euler buckling theory. CNT buckling force should have a relation with cantilever spring force too. Some energy is lost due to buckling of the nanotube and friction among nanotube layers.

Definition of set point is important in tapping mode for an appropriate application. The cantilever position should be set in smaller separations than the free vibration amplitude in order that the control system can hold the cantilever in a constant distance from the sample. In addition the larger the set point is, the better application is gotten. This is because of the reduction of the probability of impacts to steps and slopes and also the more sensitivity to the changes of distance. Set point choice also should be done regarded to buckling stiffness and CNT length. It is proper that the separation be in a range where the nanotube buckles a little. This increases the stability of images. The larger amount of CNT buckling increases the possibility of the CNT slide on the sample, creation of friction and increase of van der waals force created from horizontal positioning of nanotube.

For a precise imaging, proper characteristics should be chosen for the nanotube. The less the diameter of the CNT is, 
the better resolution is retained. This is because in imaging the tip of the cantilever should be small enough in order to enter into dips of surface, and if this doesn't happen because of large diameter of the tip related to that of the sample dips, just a small noise is created. Also the more the CNT length is, the better imaging of large steps and trenches will be retained. Choice of CNT length and diameter should be done regarded to stability too. SWCNT has a smaller diameter, but for having a proper buckling force it should have a smaller length, so it has less possibility for buckling. However, the discontinuous dynamic force-distance characteristics limit the applicability of short SWCNT probe tips. Researches showed that In SWCNT vibration amplitude has a bifurcation form, but in MWCNTs bifurcation is not seen[6].

Also in noncontact mode increasing the CNT length, causes the possibility of the adherence of cantilever and CNT to the surface[9]. This decreases the range of proper application distances. In addition increase of vertical alignment of nanotube to surface causes similar effect and so in non-contact mode shorter nanotubes with less vertical alignment angles are better. This is not so important in tapping-mode, because there is enough energy for tapping and return motion in tapping-mode.

\section{Conclusions}

Tapping-mode atomic force microscopy is an advanced method for imaging in nano scale. Carbon nanotubes cause increasing imaging resolution because of their high aspect ratio. For gaining a high resolution, AFM dynamics should be studied precisely and in this paper we tried to study the effects of attaching a CNT on the tip of an AFM. The results show that CNT tip separation from sample has the most effect on vibration behavior of AFM cantilever and the separation of probe has less effect. This is natural because of the range of the effects of intermolecular forces. Intermolecular forces show their effect in low distances, so van der Waals force is modelled by a sphere with diameter equal to the diameter of nanotube.

Decreasing the CNT length causes increasing of the buckling critical force and increasing of resonance frequency. Increase of the CNT diameter, causes increase of the resonance frequency. The CNT angle is not so effective in small ranges, but greater tilt angles cause easier buckling and bending and so having greater vibration amplitude. Increasing buckling critical force causes increasing the resonance frequency. In stiffer nanotubes which have larger critical buckling force, tapping is possible with just a little amount of buckle in the nanotube. This interesting characteristic, besides keeping touch between the nanotube and the sample, which is a base specification in tapping-mode AFM gives the amplitude equal to the separation and so causes gaining more stability. Using stiffer nanotubes removes the possibility of buckling in the range of the frequencies around natural frequency of the cantilever. Although greater force is transferred to the sample in comparison to nanotubes with less critical buckling force, but instability due to nanotube buckling is reduced too.

Totally in larger set-points and in stiffer nanotubes the amount of buckling is reduced and this can reduce the instabilities due to nanotube buckling.

\section{Appendix}

The shape functions of the beam elements are:

$$
N=\left[\begin{array}{llll}
N_{1}(x) & N_{2}(x) & N_{3}(x) & N_{4}(x)
\end{array}\right]
$$

Where

$$
\begin{gathered}
N_{1}(x)=1-3\left(\frac{x}{L}\right)^{2}+2\left(\frac{x}{L}\right)^{3} \\
N_{2}(x)=\frac{x}{L}-2\left(\frac{x}{L}\right)^{2}+\left(\frac{x}{L}\right)^{3} \\
N_{3}(x)=3\left(\frac{x}{L}\right)^{2}-2\left(\frac{x}{L}\right)^{3} \\
N_{4}(x)=-\left(\frac{x}{L}\right)^{2}+\left(\frac{x}{L}\right)^{3}
\end{gathered}
$$

Potential and kinetic energy can be defined as functions of lateral displacement like (A.6) and (A.7) or as functions of the element nodal displacement vector like (A.8) and (A.9).

$$
\begin{gathered}
P E=\frac{1}{2} \int_{0}^{L} E I\left(\frac{\partial^{2} w}{\partial x^{2}}\right)^{2} d x \\
K E=\frac{1}{2} \int_{0}^{L} \rho A\left(\frac{\partial w}{\partial t}\right)^{2} d x \\
P E=\frac{E I}{2} \int_{0}^{L} W^{T}\left(\frac{\partial^{2} N^{T}}{\partial x^{2}}\right)\left(\frac{\partial^{2} N}{\partial x^{2}}\right) W d x=\frac{1}{2} W^{T} K W \\
K E=\frac{\rho A}{2} \int_{0}^{L}\left(\dot{W}^{T} N^{T}\right)(N \dot{W}) d x=\frac{1}{2} \dot{W}^{T} M \dot{W}
\end{gathered}
$$

The governing equation can be written as

$$
\begin{gathered}
\frac{\partial}{\partial t}\left(\frac{\partial(K E-P E)}{\partial \dot{W}_{i}}\right)-\frac{\partial(K E-P E)}{\partial W_{i}}=F_{i}, \\
\quad i=1,2,3,4
\end{gathered}
$$

So mass and stiffness element matrices are

$$
\begin{gathered}
M_{e}=\frac{\rho L_{e} A}{420}\left(\begin{array}{cccc}
156 & 22 L_{e} & 54 & -13 L_{e} \\
22 L_{e} & 4 L_{e}{ }^{2} & 13 L_{e} & -3 L_{e}{ }^{2} \\
54 & 13 L_{e} & 156 & -22 L_{e} \\
-13 L_{e} & -3 L_{e}{ }^{2} & -22 L_{e} & 4 L_{e}{ }^{2}
\end{array}\right) \\
K_{e}=\frac{E I}{L_{e}{ }^{3}}\left(\begin{array}{cccc}
12 & 6 L_{e} & -12 & 6 L_{e} \\
6 L_{e} & 4 L_{e}{ }^{2} & -6 L_{e} & 2 L_{e}{ }^{2} \\
-12 & -6 L_{e} & 12 & -6 L_{e} \\
6 L_{e} & 2 L_{e}{ }^{2} & -6 L_{e} & 4 L_{e}{ }^{2}
\end{array}\right)
\end{gathered}
$$

In Newmark method the time interval is divided into smaller time steps $(\Delta t)$ and in each step, it is assumed that the acceleration of each node is constant.

$$
M \ddot{W}(t)+C \dot{W}(t)+K W(t)=F(t)
$$

Assume $\ddot{W}(t)=$ cte 


$$
\begin{gathered}
W(t+\Delta t)=W(t)+\dot{W}(t) \Delta t+\ddot{W}_{\text {average }}(t) \frac{\Delta t^{2}}{2} \\
\dot{W}(t+\Delta t)=\dot{W}(t)+\ddot{W}(t) \Delta t \\
M \ddot{W}(t+\Delta t)+C \dot{W}(t+\Delta t)+K W(t+\Delta t)=F(t+\Delta t) \\
\ddot{W}_{\text {average }}=\frac{\ddot{W}(t+\Delta t)+\ddot{W}(t)}{2}
\end{gathered}
$$

\section{REFERENCES}

[1] H. Dai, J.H. Hafnter, A. G. Rinzler, D. T. Colbert, R. E. Smalley, "Nanotubes as nanoprobes in scanning probe microscopy", Nature 384 (1996) 147-15

[2] L. Chen, Ch. L. Cheung, P. D. Ashby, Ch.M. Lieber, "Single-Walled Carbon Nanotube AFM Probes: Optimal imaging resolution of nanoclusters and biomolecules in ambient and fluid environments", Nano Letters, Vol. 4, No. 9 (2004) 1725-1731

[3] M. C. Strus, A. Raman, C. V. Nguyen, "Imaging artefacts in atomic force microscopy with carbon nanotube tips", Nanotechnology 16 (2005) 2482-2492

[4] E. Lee, "An integrated system of microcantilever arrays with carbon nanotube tips for imaging, sensing, and 3D nanomanipulation: Design and control", Sensors and Actuators, A134 (2007) 286-295

[5] S. I. Lee, S. W. Howell, A. Raman, R. Reifenberger, "Nonlinear dynamics of microcantilevers in tapping mode atomic force microscopy: A comparison between theory and experiment”, Physical Review, B66 (2002) 115409

[6] S. I. Lee, S.W. Howell, A. Raman, R. Reifenberger, C.V. Nguyen, M. Meyyappan, "Complex dynamics of carbon nanotube probe tips", Ultramicroscopy 103 (2005) 95-102

[7] S. I. Lee, S.W. Howell, A. Raman, R. Reifenberger, C. V. Nguyen, M. Meyyappan, "Nonlinear tapping dynamics of multi-walled carbon nanotube tipped atomic force microcantilevers", Nanotechnology 15 (2004) 416-421

[8] C. V. Nguyen, K.J. Chao, R. M. D. Stevens, L. Delzeit, A. Cassell, J. Han, M. Meyyappan, "Carbon nanotube tip probes: stability and lateral resolution in scanning probe microscopy and application to surface science in semiconductors", Nanotechnology 12 (2001) 363-367

[9] E. S. Snow, P.M. Campbell and J.P. Novak, "Single-wall carbon nanotube atomic force microscope probes", Applied Physics Letters, Vol. 80, No. 11 (2002) 2002-2004

[10] B. Bhushan, B. Galasso, C. Bignardi, C. V Nguyen, L. Dai and L. Qu; "Adhesion, friction and wear on the nanoscale of MWNT tips and SWNT and MWNT arrays", Nanotechnology 19 (2008) 125702

[11] H. Lua, J. Goldmana, F. Dinga, Y. Suna, M.X. Pulikkatharab, V.N. Khabasheskub, B.I. Yakobsona, J. Loua, "Friction and adhesion properties of vertically aligned multiwalled carbon nanotube arrays \& fluoro-nanodiamond films", Carbon 46 (2008) 12941301

[12] H. W. Yap, R. S. Lakes, and R. W. Carpick, "Mechanical instabilities of individual multiwalled carbon nanotubes under cyclic axial compression", Nano Letters, Vol. 7, No. 5 (2007) 1149-1154

[13] D. V. Hutton, "Fundamentals of finite element analysis", The McGraw-Hill companies (2004)

[14] S. G. Kelly, "Fundamentals of mechanical vibrations", $2^{\text {nd }}$ Ed.

[15] J. L. Meriam, L. G. Kraige; "Engineering Mechanics"; $5^{\text {th }}$ Ed; John Wiley \& Sons, Inc.

[16] Y. Song, B. Bhushan, "Finite-element vibration analysis of tapping-mode atomic force microscopy in liquid", Ultramicroscopy 107 (2007) 1095-1104

[17] R. Garcia, R. Perez "Dynamic atomic force microscopy methods", Surf. Science, Rep. 47 (2002) 197-301 